Psychother Psychosom 2020;89:56-58

DOI: $10.1159 / 000503904$

\section{Placebo Effects of Nurses' Communication alongside Standard Medical Care on Pain and Other Outcomes: A Randomized Controlled Trial in Clinical Tonsillectomy Care}

\author{
Liesbeth M. van Vliet ${ }^{\mathrm{a}, \mathrm{b}}$ Marc B. Godfried ${ }^{\mathrm{a}}$ Gerard W. van Deelen ${ }^{\mathrm{c}}$ \\ Menno Kaunang ${ }^{\mathrm{b}}$ Ted J. Kaptchuk ${ }^{\mathrm{d}}$ Sandra van Dulmen ${ }^{\mathrm{b}, \mathrm{e}, \mathrm{f}}$ \\ Bram Thiel ${ }^{\mathrm{a}}$ Jozien M. Bensing ${ }^{\mathrm{b}}$ \\ a Department of Anesthesiology, OLVG Hospital, Amsterdam, \\ The Netherlands; ${ }^{b}$ Department of Communication, NIVEL \\ (Netherlands institute for health services research), Utrecht, \\ The Netherlands; ' Department of ENT, OLVG Hospital,

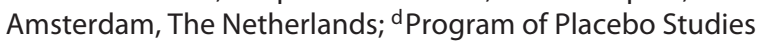 \\ and the Therapeutic Encounter, Beth Israel Deaconess Medical \\ Center, Harvard Medical School, Boston, MA, USA; ${ }^{~}$ Radboud \\ university medical center, Radboud Institute for Health Sciences, \\ Department of Primary and Community Care, Nijmegen,

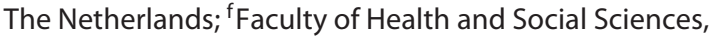 \\ University of South-Eastern Norway, Drammen, Norway
}

Patient outcomes are not only influenced by medical technical interventions, but also by the context in which these interventions are provided. Such effects are called placebo effects [1]. They are elicited via several mechanisms, and can impact patient outcomes, most notably pain. The best-known mechanism is expectancy, whereby manipulating patients' expectations impacts pain $[2,3]$. A lesser-known mechanism is empathy $[4,5]$.

A relatively unexplored, promising perspective is the role of clinician communication in eliciting placebo effects. A landmark study found that placebo-acupuncture delivered by a confident, empathic clinician produced significant changes in irritable bowel syndrome patients' outcomes [5]. In subsequent experimental studies, we disentangled the effects of clinician expectancy (affecting cognitive outcomes, e.g., expected pain treatment outcome) and empathy (affecting affective outcomes, e.g., anxiety) with most positive effects when expectancy and empathy were combined [6, 7]. To strengthen the clinical applicability of placebo effects, we need to know whether similar effects will be found in clinical care alongside standard evidence-based medical interventions. This study aimed to determine the separate and combined effects of: (i) expectancy-manipulation (standard vs. enhanced) and (ii) empathy-manipulation (standard vs. enhanced) through nurses' communication alongside standard medical care on clinical tonsillectomy patients' outcomes.

Detailed information about the intervention and methods can be found in the published study protocol [8] and the supplemen- tary material (see www.karger.com/doi/10.1159/000503904 for all online suppl. material). A four-arm $(2 \times 2$ design $)$ single-blind randomized controlled trial was conducted at 2 daycare nursing wards. In total, 128 patients ( $>18$ years, scheduled for tonsillectomy in daycare, who could speak/understand Dutch and had no mental incapacity) were recruited and randomized to one of four groups (group 1: expectations+/empathy+; group 2: expectations-/empathy+; group 3: expectations+/empathy-; group 4: expectation-/empathy-).

The intervention consisted of a protocolled communication manipulation on top of the standard analgesic treatment protocol and daily routine care. For each group, daycare ward nurses manipulated their communication (either standard or enhanced expectations and empathy) during the patients' stay at the daycare ward (pre- and post-operation, day 1) and during the telephone consultation day post-discharge (day 2). Other clinicians standardized their communication. Nurses were trained in the delivery of the intervention using a half-day training course and several booster sessions. The research team was (onsite) available for feedback and information cards/posters were displayed. Norms and values of acceptable behavior were not crossed.

Within the expectancy manipulation the expectation that the pain medication would work very well was either not given (standard condition, example sentence: "This is your (pain) medication") or was given (enhanced condition, example sentence: “This pain medication is known for working very well"). Within the empathy manipulation a (non-)verbal empathic atmosphere was either not created (standard condition, example behaviors: reacting with standard empathy to patients' cues/concerns, not paying extra interest in the patient as a person) or was created (enhanced condition, example behaviors: reacting extra empathically to patients' cues/concerns, showing extra interest in the patient as a person).

The main analyses (detailed information is provided in the supplementary material) using STATA 14.0 consisted of ANCOVAs to determine the main and interaction effects of expectancy and empathy on patients' (pain) outcomes. Our results (detailed information is provided in the supplementary material) demonstrated that our sample was young (mean age 28 years), female (67\%), and highly educated (63\%; online suppl. Table 2$)$. The manipulations were perceived as being in the right direction, but this remained non-significant (expectancy standard: mean $=6.63$; high: mean $=$ $7.18, t=-1.62, p=0.11$, range $0-10$; and empathy standard: mean $=39.70, \mathrm{SD}=9.33$; high: mean $=42.63, t=-1.56, p=0.12$, range 10-50). In contrast, the audio-recordings of nurse-patient interactions showed that $82 \%$ of the expectancy and $68 \%$ of the empathy manipulations were successfully displayed. Anxiety towards the operation had a significant effect on day 1 maximum pain $(\beta=0.14, p=0.01)$, and was included as a centered covariate in the analyses. ANCOVA analyses showed no significant main and interaction effects of the manipulations on patients' perceived

\section{KARGER}

(C) 2019 S. Karger AG, Basel 
Table 1. Main effects of expectancy and empathy on outcomes

\begin{tabular}{|c|c|c|c|c|c|c|}
\hline & \multicolumn{3}{|c|}{ Expectancy } & \multicolumn{3}{|c|}{ Empathy } \\
\hline & $F$ & SS & $p$ & $F$ & SS & $p$ \\
\hline \multicolumn{7}{|l|}{ Pain primary } \\
\hline Max. pain day 1 & 0.63 & 1.97 & 0.43 & 0.91 & 2.83 & 0.34 \\
\hline Max. pain day 2 & 0.00 & 0.01 & 0.96 & 0.33 & 1.05 & 0.57 \\
\hline Pain day 3 & 0.95 & 2.89 & 0.33 & 1.46 & 4.43 & 0.23 \\
\hline \multicolumn{7}{|l|}{ Pain secondary } \\
\hline Pain expectation & 0.17 & 0.50 & 0.68 & 0.97 & 2.81 & 0.33 \\
\hline Pain improvement expectation & 2.50 & 831.80 & 0.12 & 0.25 & 82.30 & 0.62 \\
\hline Pain evaluation & 5.58 & 23.98 & 0.02 & 0.14 & 0.59 & 0.71 \\
\hline \multicolumn{7}{|l|}{ Psychological } \\
\hline Anxiety & 0.27 & 11.43 & 0.60 & 2.28 & 94.99 & 0.14 \\
\hline Positive mood & 0.00 & 0.00 & $>0.99$ & 0.02 & 1.40 & 0.88 \\
\hline Negative mood & 1.10 & 54.17 & 0.30 & 0.00 & 0.12 & 0.96 \\
\hline Satisfaction & 0.25 & 0.70 & 0.62 & 0.15 & 0.40 & 0.70 \\
\hline \multicolumn{7}{|l|}{ Other outcomes } \\
\hline OBAS day 2 & 0.01 & 0.13 & 0.93 & 0.05 & 0.75 & 0.83 \\
\hline OBAS day 3 & 1.85 & 29.80 & 0.18 & 1.58 & 25.40 & 0.21 \\
\hline General quality & 2.80 & 3.07 & $<0.10$ & 0.14 & 0.15 & 0.71 \\
\hline Recommendation & 1.43 & 2.56 & 0.24 & 0.80 & 1.43 & 0.38 \\
\hline
\end{tabular}

All analyses were controlled for (centered) effects of anxiety towards the operation. Interaction effects were insignificant and thus eliminated from the model. Bold $p$ values are considered significant.

pain (Table 1; online suppl. Table 4). Nurses' enhanced expression of the pain medications' effectiveness did not lower pain levels on day $1(p=0.43)$, day $2(p=0.96)$, or day $3(p=0.33)$, and nurses' enhanced expression of empathy did not lower pain levels on day $1(p=0.34)$, day $2(p=0.57)$, or day $3(p=0.23)$. The pain protocol was well adhered to and received analgesia did not differ between conditions, apart from paracetamol day $3(F=0.08$; online suppl. Table 5). Regarding our secondary outcomes (Table 1; online suppl. Table 4), neither expectancy nor empathy influenced patients' pain expectations for the upcoming days $(p=0.68 ; p=0.33)$ or patients' pain improvement expectations after receiving medication $(p=0.12 ; p=0.62)$. On day 3 , patients receiving high expectations thought their post-operative pain had been better than expected $(p=0.02)$, which was unaffected by empathy $(p=0.71)$. Expectations and empathy did not significantly affect patients' psychological outcomes: anxiety $(p=0.60, p=0.14)$, positive mood $(p>0.99, p=0.88)$, negative $\operatorname{mood}(p=0.30, p=0.96)$, and satisfaction $(p=0.62, p=0.70)$. Patients' overall benefit of analgesia was not influenced by expectations and empathy (day 2: $p=0.93, p=$ 0.83 ; day 3 : $p=0.18, p=0.21$ ). When nurses' expressed high painrelieving expectations, patients tended to rate the quality of the received care higher $(p<0.10)$, which was unaffected by empathy $(p=0.71)$. The likelihood to recommend this hospital remained unaffected by expectancy and empathy $(p=0.24, p=0.38)$.

Although these results were not as anticipated based on previous evidence $[2,3,6,7,9]$, we need to interpret them in light of the choppy waters of the clinical routine care reality we entered with this study. This included methodological complexities (e.g., effects to be found from communication alongside standard care might be smaller than anticipated; a pool of nurses delivering the intervention) and limitations (e.g., manipulations not being successfully perceived; missing data) that need to be overcome in future studies. Simultaneously, we should not dismiss the potential positive placebo effects of communication we did find, i.e., positive expectations led to better evaluations of perceived pain and received care. We hope our study will act as a starting point for further studies in this important yet fragile research field, disentangling the potential effects of expectancy and empathy on clinical patients' outcomes alongside standard care. In the meantime, raising positive yet realistic expectations in an empathic manner is recommended [10]. It is harmless, time-efficient, and has the potential to improve at least some patients' outcomes for the better.

\section{Acknowledgements}

We thank Peter Spreeuwenberg for his help with the data analysis and power calculation. We thank the clinicians working at the daycare ward, the operation theater, the PACU, and at the ENT and Anesthesia department for their help in facilitating the study logistics. We especially thank the daycare ward nurses for delivering the intervention. We thank Stephanie Immerzeel, Veerle van Laarhoven, Jill Damen, Melissa Koudijs, Kirsten Boothby, Renske Hoogstraten, and Joris Kromhout for their help in the data collection, and Janneke Noordman for her help in supervising. We thank Irving Kirsch for his help with setting up the study design. We thank all patients for participating. We would like to thank the OLVG data information service for their technical assistance in retrieving necessary information from participants' medical records. 
Statement of Ethics

All participants gave their written informed consent. The study received formal ethical approval from the ethics committee of the UMC Utrecht (No. 16/144, NL55225.041) and the institutional review board of the participating hospital (No. WO 16.506). It is registered at the Dutch Trial Register (Registration No. NTR5994), which is the Primary Registry in the Netherlands.

\section{Disclosure Statement}

The authors have no conflicts of interest to declare.

\section{Funding Sources}

The study was primarily funded by the Spinoza Prize from the Dutch Research Council awarded to J.M.B. L.M.v.V. was partly funded by a grant from the Foundation for the Science of the Therapeutic Encounter (F-STE). The study was partly funded by the Scientific Foundation of the OLVG Hospital Amsterdam (No. 16.058). The funders had no role in the study design, collection, analysis and interpretation of the data, in writing the report, and in the decision to submit the report for publication. All authors are independent from the funders and had full access to all of the data in the study and can take responsibility for the integrity of the data and the accuracy of the data analysis.

\section{Author Contributions}

J.M.B., L.M.v.V., M.B.G., G.W.v.D., T.J.K., S.v.D., and B.T. were involved in the planning of the study. All authors were involved in the conduct of the study and in reporting of the work. L.M.v.V. is the guarantor. The corresponding author attests that all listed authors meet authorship criteria and that no others meeting the criteria have been omitted.

\section{References}

1 Finniss DG, Kaptchuk TJ, Miller F, Benedetti F. Biological, clinical, and ethical advances of placebo effects. Lancet. $2010 \mathrm{Feb}$;375(9715):686-95.

2 Peerdeman KJ, van Laarhoven AI, Keij SM, Vase L, Rovers MM, Peters ML, et al. Relieving patients' pain with expectation interventions: a metaanalysis. Pain. 2016 Jun; 157(6):1179-91.

3 Mistiaen P, van Osch M, van Vliet L, Howick J, Bishop FL, Di Blasi Z, et al. The effect of patient-practitioner communication on pain: a systematic review. Eur J Pain. 2016 May;20(5):675-88.

4 Bensing JM, Verheul W. The silent healer: the role of communication in placebo effects. Patient Educ Couns. 2010 Sep;80(3):293-9.

5 Kaptchuk TJ, Kelley JM, Conboy LA, Davis RB, Kerr CE, Jacobson EE, et al. Components of placebo effect: randomised controlled trial in patients with irritable bowel syndrome. BMJ. 2008 May;336(7651):9991003.

6 Verheul W, Sanders A, Bensing J. The effects of physicians' affect-oriented communication style and raising expectations on analogue patients' anxiety, affect and expectancies. Patient Educ Couns. 2010 Sep;80(3): 300-6.

7 van Osch M, van Dulmen S, van Vliet L, Bensing J. Specifying the effects of physician's communication on patients' outcomes: A randomised controlled trial. Patient Educ Couns. 2017 Aug;100(8):1482-9.

8 van Vliet LM, van Dulmen S, Thiel B, van Deelen GW, Immerzeel S, Godfried MB, et al. Examining the effects of enhanced provider-patient communication on postoperative tonsillectomy pain: protocol of a randomised controlled trial performed by nurses in daily clinical care. BMJ Open. 2017 Nov;7(11):e015505.

9 Benedetti F, Maggi G, Lopiano L, Lanotte M, Rainero I, Vighetti S, et al. Open versus hidden medical treatments: the patient's knowledge about a therapy affects the therapy outcome. Prev Treat. 2003;6(1):1a.

10 Evers AW, Colloca L, Blease C, Annoni M, Atlas LY, Benedetti F, et al. Implications of placebo and nocebo effects for clinical practice: expert consensus. Psychother Psychosom. 2018;87(4):204-10. 